\title{
Flare coupled metal parallel-plate waveguides for high resolution terahertz time-domain spectroscopy
}

\author{
Michael Theuer, ${ }^{1,2}$ S. Sree Harsha, ${ }^{1}$ and D. Grischkowsky ${ }^{1, a)}$ \\ ${ }^{1}$ School of Electrical and Computer Engineering, Oklahoma State University, Stillwater, \\ Oklahoma 74078, USA \\ ${ }^{2}$ Department of Terahertz Measurement and Systems, Fraunhofer Institute for Physical Measurement \\ Techniques, 67663 Kaiserslautern, Rhineland-Palatinate, Germany
}

(Received 12 July 2010; accepted 14 October 2010; published online 3 December 2010)

\begin{abstract}
We report on a new coupling scheme for high resolution terahertz spectroscopy of microcrystalline films using parallel-plate waveguides. Metal flares are used to couple the terahertz radiation into and out of the waveguide. Very good coupling ratios as high as $35 \%$ at $1 \mathrm{THz}$ from a collimated free-space beam into a subwavelength gap are obtained. This microwave approach is compared in terms of coupling ratio and spectral characteristics to the established technique of quasioptic coupling to parallel-plate waveguides using silicon lenses. Various samples at room and cryogenic temperatures are measured to show the capabilities of flare coupling for high resolution terahertz spectroscopy. ㅇ 2010 American Institute of Physics. [doi:10.1063/1.3516307]
\end{abstract}

\section{INTRODUCTION}

Terahertz (THz) (far-infrared) absorption spectroscopy is an important technique for the characterization of molecules and crystals. The probed transitions are either short range (rotational ${ }^{1}$ or vibrational ${ }^{2}$ ), or long distance interactions such as lattice vibrations, ${ }^{3}$ bending modes or van-der-Waals interactions. ${ }^{4}$ Depending on the aggregate state and the particular sample, the characteristic frequencies of the transitions can be in the THz frequency range. This band of the electromagnetic spectrum covers frequencies between 100 $\mathrm{GHz}$ and $10 \mathrm{THz}$, corresponding to vacuum wavelengths between $3 \mathrm{~mm}$ and $30 \mu \mathrm{m}$. To address a large fraction of the $\mathrm{THz}$ band, $\mathrm{THz}$ time-domain spectroscopy (THz-TDS) (Ref. 5) has proven to be a useful tool if broadband radiation between 0.1 and $4 \mathrm{THz}$ is needed.

To obtain an unambiguous spectral fingerprint of molecular solids under study at $\mathrm{THz}$ frequencies, special care needs to be given to grow single crystals which is often a complicated process. ${ }^{3}$ Alternatively, for the pellet technique, which involves measuring a diluted compressed powder of the substance, ${ }^{6}$ the spectral resolution is limited by many broadening mechanisms. These are due to the heterogeneous distribution of microcrystals within the pellet and their random orientation with respect to the incident $\mathrm{THz}$ radiation, resulting in broadened lineshapes of molecular absorption.

Melinger $e t a l^{7}$ have recently demonstrated a THz spectroscopic technique based on metal parallel-plate waveguides (PPWGs). ${ }^{8}$ A thin polycrystalline film of a sample is dropcast on one of the metal plates comprising the PPWG and then probed using the transverse electromagnetic (TEM) mode of the metal PPWG. This technique only requires microgram sample quantities due to the spatial confinement, and it also facilitates the measurement of high resolution $\mathrm{THz}$ absorption spectra of the samples. Significant reduction in inhomogeneous broadening is obtained, due to the formation of high

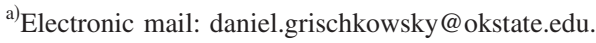

quality microcrystals which are highly oriented to the metal surface. Further reduction in homogeneous (thermal) broadening is obtained by cooling the sample to cryogenic temperatures. A detailed description of sample preparation is presented in Ref. 3.

In this article, we will discuss the possibilities of flare coupling to PPWGs as a simplification of the developed quasioptical technique using silicon lenses. This easy-to-use and low-cost alternative can match the performance (coupling ratio, spectral resolution, required sample quantity) of the PPWG with lens coupling. We will first describe WG THzTDS with quasioptical coupling, then introduce flared coupling, and finally compare the two approaches.

\section{THz WG SPECTROSCOPY}

The experimental setup consists of three main components: The THz-TDS system, the WG, and the sample. The THz-TDS system is based on the coherent sampling of the $\mathrm{THz}$ transients using photoconductive switches gated by femtosecond laser pulses ${ }^{5}$ (for setup see Fig. 1). The $\mathrm{THz}$ pulses are generated by a photoconductive antenna (Tx), collected via a silicon lens, collimated by a pair of parabolic

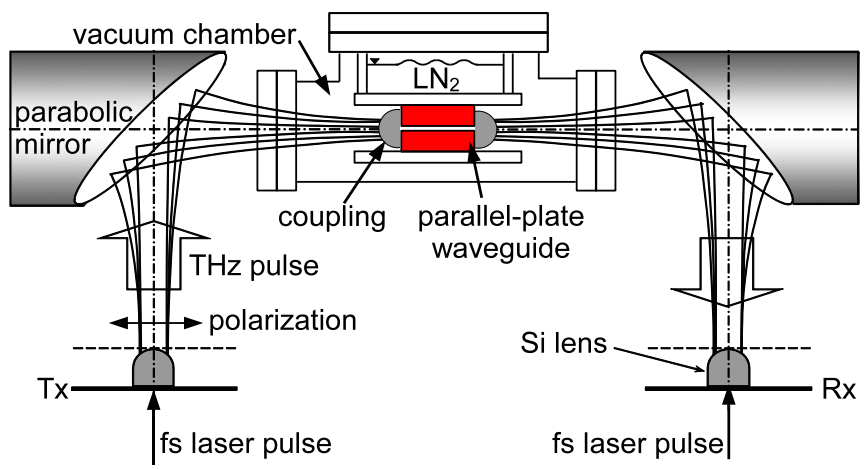

FIG. 1. (Color online) Beam path of the THz-TDS system. In the waist of the collimated beam, the PPWGs are measured between the two parabolic mirrors. Here a PPWG is shown with silicon lens coupling. 
a)
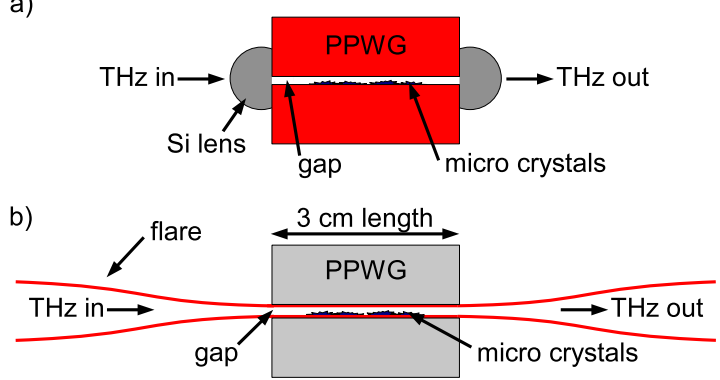

FIG. 2. (Color online) Cross-sectional illustration of the two types of coupling schemes used for PPWGs. (a) PPWG with coupling using silicon lenses. (b) Same WG with flared coupling using copper shim.

mirrors, and then detected by a photoconductive antenna $(\mathrm{Rx})$. The WG assembly is placed at the frequency dependent beam-waist $(9 \mathrm{~mm}$ at $1 \mathrm{THz})$ obtained between the two parabolic mirrors. ${ }^{9}$ The $\mathrm{THz}$ radiation is linearly polarized in the plane of projection of Fig. 1.

A standard THz PPWG (Ref. 8) is shown in Fig. 2(a). It consists of two polished copper plates $(1 \times \mathrm{w} \times \mathrm{h}=30 \times 28$ $\times 9.5 \mathrm{~mm}^{3}$ ) with a separation of less than half the $\mathrm{THz}$ wavelength. Spacers between the two screwed together plates maintain the gap at a defined value, typically $50 \mu \mathrm{m}$. The PPWG is oriented with the THz electric field perpendicular to the metal surfaces to support the TEM mode. For our typically used $50 \mu \mathrm{m}$ PPWG spacing, THz radiation polarized parallel to the WG plates will not be transmitted for frequencies below the TEl cut-off frequency of $3 \mathrm{THz}$. In addition, our $\mathrm{THz}$ receiver is sensitive only to polarization along the antenna direction. The collimated $\mathrm{THz}$ beam is focused into the subwavelength gap and recollimated after passage through the WG, using a pair of planocylindrical silicon lenses, in contact and aligned to the center gap of the PPWG. The lenses' geometry is optimized for broadband high transmission (radius of curvature $5 \mathrm{~mm}$, thickness of $6.56 \mathrm{~mm}$, and length of $15 \mathrm{~mm}$.). This type of coupling is efficient, however, it suffers from the inherent Fresnel losses at the four interfaces between air and silicon $\left[\mathrm{n}_{\mathrm{Si}}=3.42\right.$ (Ref. 10)] causing an amplitude loss of $50 \%$. Beam truncation at the lens edges and the gap [elliptical THz spotsize after the Si lens with minor axis of $150 \mu \mathrm{m}$ (Ref. 11)], modal mismatch and Ohmic losses in the WG contribute further losses. The typical measured amplitude coupling ratios are roughly $20 \%$ compared to the theoretically possible optimal value of $32 \% .^{11}$

\section{FLARED COUPLING}

As the $\mathrm{THz}$ frequency band is located between the microwaves and the infrared, coupling schemes known in both neighboring domains can be used. The quasioptical method using lenses is the infrared approach. ${ }^{9}$ In this article, we apply the well known microwave approach based on curved metal surfaces (horn antenna) to couple linearly polarized $\mathrm{THz}$ radiation into PPWGs (Ref. 12) and compare its performance to the silicon lens coupled PPWGs for high resolution WG THz-TDS. As we have shown recently, curved flares made out of metal shim can be efficiently used to couple THz radiation. ${ }^{13}$ In the quasioptical approach, the metal sur-

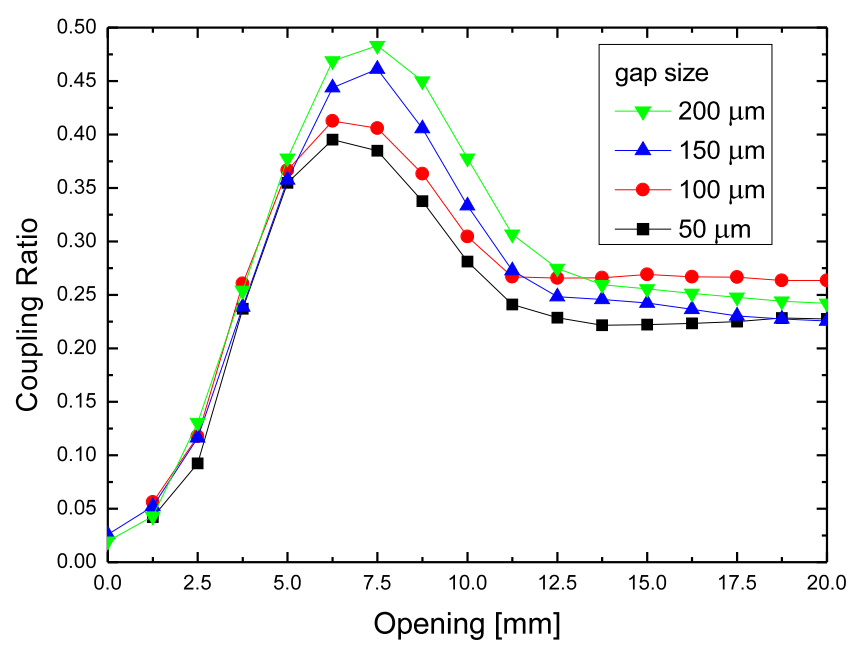

FIG. 3. (Color online) Measured amplitude coupling ratios for a flare of 15 $\mathrm{cm}$ length with different gap sizes. The transmission can be maximized by adjusting the flare opening for the best adiabatic coupling.

faces would just reflect the $\mathrm{THz}$ waves. But, if the changes in the beam extent are introduced adiabatically, ${ }^{14}$ which denotes slowly with respect to the wavelength, the reflection losses can be minimized and propagation in the forward direction is maintained.

The production of the flares is simple and fast. Using a paper cutter, $15 \mathrm{~mm}$ wide strips are cut from a $9 \mathrm{~cm}$ diameter, $15 \mathrm{~cm}$ wide roll of standard commercial $100 \mu \mathrm{m}$ thick copper shim. The strips are then slightly pressed and rolled using a metal cylinder to make them flat and to remove bends. For experiments in the $\mathrm{THz}$ range the surface quality of the commercial shim is of optical grade.

To demonstrate the efficiency of this type of coupling, a metal flare with overall length of $15 \mathrm{~cm}$ was measured as a function of opening distance for different gap widths (see Fig. 3). As discussed in Ref. 13, such a flare adiabatically compresses the linearly polarized $\mathrm{THz}$ beam into the gap and then guides the adiabatic expansion of the output beam. Two $25 \mathrm{~mm}$ diameter circular rods compress the copper shims together in the center [see Fig. 1(b), Ref. 13], while the gap is kept open by the mechanical spacers outside of the $\mathrm{THz}$ beam. Consequently, the coupling can be investigated independent of the WG or sample losses.

There is an optimal flare opening for the best coupling ratio, defined by the peak-to-peak amplitude transmission in the time domain with and without the flare assembly in the beam. Independent of the gap size, all flares show the best performance at a full opening of slightly above $5 \mathrm{~mm}$. This distance is defined by an interplay between the adiabatic shape of the curvature (minimal reflection losses) and the incident beam size (minimal beam truncation). As the transition of the flare remains smooth for subwavelength gaps, no large reduction in the coupling ratio is seen for narrower gaps. The obtained coupling ratios are $48 \%$ for a $200 \mu \mathrm{m}$ gap and $39 \%$ for $50 \mu \mathrm{m}$, respectively. This may be useful for further sensitivity increase if the gap size has to be reduced for weakly absorbing samples. It should be mentioned that the alignment can be optimized for highest peak ampli- 


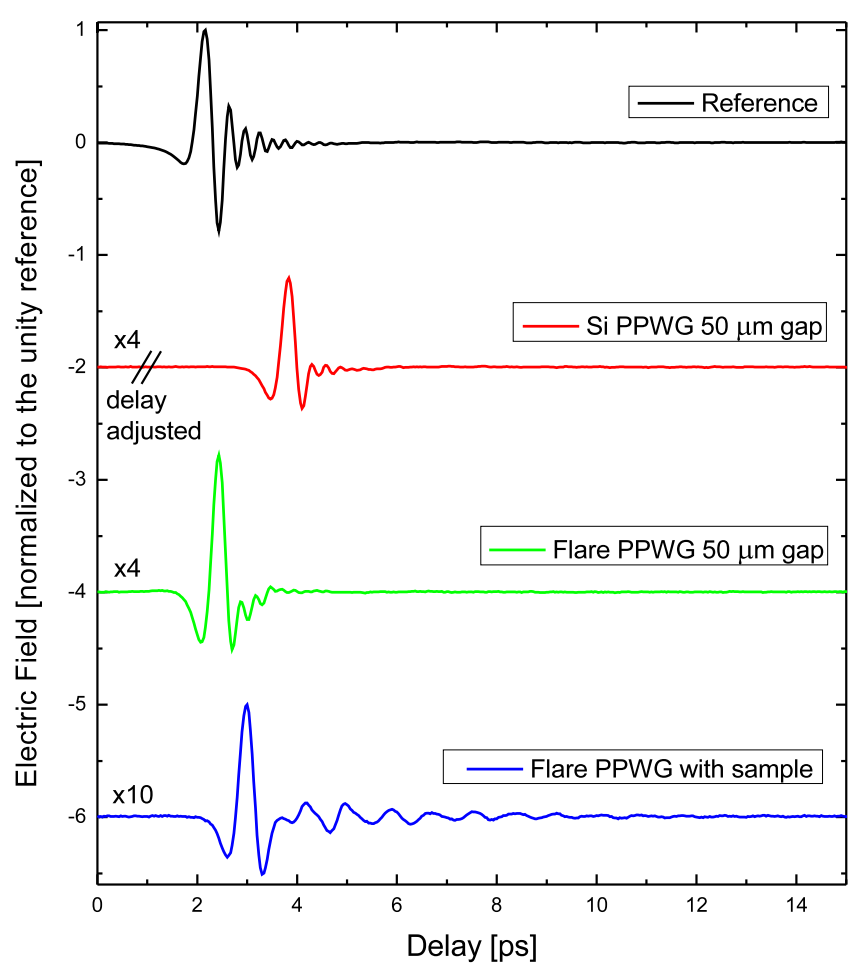

FIG. 4. (Color online) Measured $\mathrm{THz}$ transients (top-bottom): reference pulse (normalized to unity) in dry air chamber; pulse after propagation through the Si lens PPWG; pulse after propagation through the flare PPWG; TCNQ sample in the flare PPWG. The pulse shape remains undistorted by the flare coupling. Ringing caused by absorption features as well as the additional delay shows the strong interaction with the sample.

tude (values above $50 \%$ were reached) or best broadband coupling which is desirable for spectroscopic applications.

This type of coupling is nearly distortionless as seen in Fig. 4. The transmitted THz pulses are shown for the reference (empty chamber with dry air), a standard PPWG with a $50 \mu \mathrm{m}$ gap using Si lenses, a flare PPWG (also $50 \mu \mathrm{m}$ gap) and the same flare PPWG with a sample (dropcast TCNQ at room temperature). Note that the Si-PPWG and the flare PPWG pulse were upscaled by a factor of 4 and the sample pulse by a factor of 10 . The delay axis for the Si-PPWG was adjusted to compensate for the relative transit time through the two Si lenses. The reference pulse is echo free out to a delay of 180 ps and broadband as can be seen by the short pulse duration and the deep minimum. Once the PPWGs are placed in the $\mathrm{THz}$ beam, the transmitted pulse amplitude drops. The reduction is larger for the Si-PPWG than for the flared PPWG. The second minimum in the pulse shape is not as pronounced due to reduction in the high frequency components. But the pulse shape is still similar to the reference for both flares. No disturbing reflections within the typical scanning distances of 150 ps delay are observed. However, the cylindrical Si lenses of the quasioptic approach cause a small reflection echo at $150 \mathrm{ps} .{ }^{15}$ By introducing the sample, the delay of the pulse increases, and the absorption causes additional ringing after the main pulse. A Fourier transform uncovers the corresponding absorption features.

A direct comparison between the spectral coupling using the metal flare and the pair of Si lenses is given in Fig. 5. It shows the frequency dependent amplitude coupling ratios

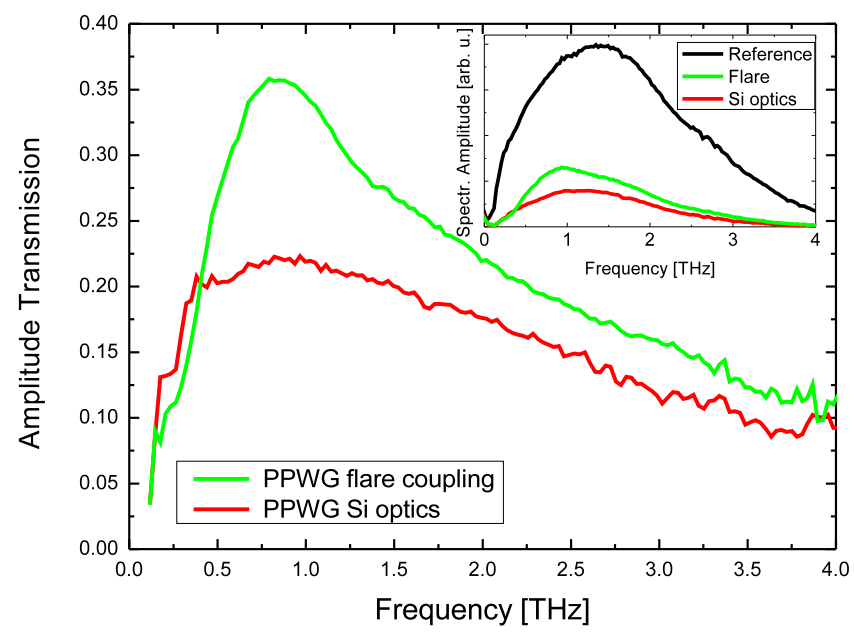

FIG. 5. (Color online) Frequency dependent coupling ratios (amplitude transmission) obtained for the two types of coupling with identical WG properties. The flare shows better coupling over the entire frequency band compared to the Si lenses. Inset: measured spectral amplitudes before division by the reference.

(amplitude transmission) for a flare (12 cm long, opening 5 $\mathrm{mm}$ ) and a standard PPWG. The same parallel plates were used for both measurements with a $50 \mu \mathrm{m}$ gap. No sample was introduced into the gap. The measurement was carried out at room temperature and in a dry air atmosphere. Unlike the measurements presented in Fig. 3, the flare did not have a linear stage to precisely control the opening. This was neither necessary nor spatially possible as the same WGs were later to be measured in the vacuum chamber for high resolution spectroscopy at cryogenic temperatures. So, the copper shim was manually formed into an appropriate funnellike shape. The spacers are located between the flares but outside of the $\mathrm{THz}$ beam. The same metal plates used for the Si-PPWG formed a rigid screwed together sandwichlike flare assembly which could be easily handled.

The measured coupling ratios of the flare are larger than for the Si optics. At $1 \mathrm{THz}$ the coupling ratio is $35 \%$ for the flared WG compared to $22 \%$ for the Si-PPWG. Also, at higher frequencies up to $4 \mathrm{THz}$ the coupling ratio remains better for the flare but the difference becomes smaller. In the inset of Fig. 5 the measured spectral amplitudes of the measured data are plotted before division by the reference spectrum. The reference has a very high signal-to-noise ratio with spectral contributions beyond $4 \mathrm{THz}$, but the coupling attenuation limits the accessible WG frequency range to 4 THz.

\section{SPECTROSCOPIC RESULTS}

Three samples, which have been characterized earlier with the Si lens coupled PPWG, were chosen to demonstrate the performance of flare coupling for high resolution spectroscopy. The organic molecules tetracyanoquinodimethane (TCNQ) (Ref. 3) and 1,2-dicyanobenzene (1,2-DCB), ${ }^{7}$ and the pharmaceutical drug salicylic acid ${ }^{16}$ were characterized. Solutions of these three samples were dropcast on the metal shim. After evaporation of the solvent a microcrystalline film remains on the surface of the copper shim (see Fig. 6). This surface is used to form the center region of the WG. An 


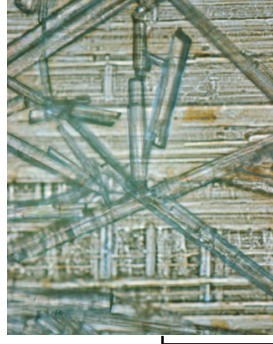

1,2-DCB

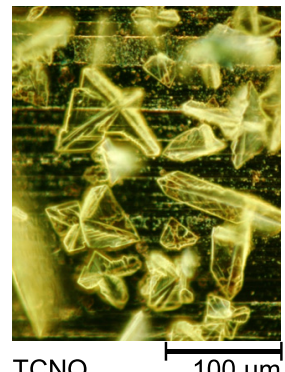

$100 \mu \mathrm{m}$

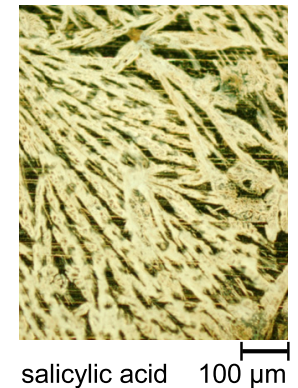

FIG. 6. (Color online) Microscope images of the dropcast samples. Individual microcrystals are located on the surface of the copper shim. Depending on the sample, different crystal geometries are formed.

identical blank copper shim forms the opposite surface of the PPWG as shown in Fig. 2(b). First the PPWGs are measured at room temperature to check the amount of applied sample and the remaining coupling ratio. Then they are inserted into the vacuum chamber and cooled down to liquid nitrogen $\left(\mathrm{LN}_{2}\right)$ temperature of $77 \mathrm{~K}$. This $\mathrm{LN}_{2}$ container and the vacuum chamber are placed in the center of the THz-TDS system as shown in Fig. 1.

Figure 7 shows the spectroscopic results for a flare coupled WG (12 cm long flare, $50 \mu \mathrm{m}$ gap) with a dropcast films of crystalline 1,2-DCB, TCNQ, and salicylic acid in comparison to the results obtained with the Si lens coupled PPWG. ${ }^{3,7,16}$ Evidence of absorption features is seen at room temperature $(295 \mathrm{~K})$ for all the samples. The absorption lines become much narrower when the samples are cooled down to $\mathrm{LN}_{2}(77 \mathrm{~K})$, demonstrating the quality and planar orientation of the microcrystals. These much sharper lines are described more precisely in terms of their line centers and line widths in Table I. For all three samples the absorption features are seen to undergo a blueshift to higher frequency with a decrease in temperature. This shift is often attributed to compression of the crystalline lattice which steepens the intermolecular potential and thereby shifts the absorption resonance to higher frequencies. The shift is very reproducible, as shown when a single line is monitored. ${ }^{17}$ In the inset the corresponding absorbance is displayed, which is calculated by a spline fit of the spectral amplitude to a reference envelope.

Table I lists the comparison between the measured absorption lines at $77 \mathrm{~K}$ for 1,2-DCB, TCNQ, and salicylic acid. WGs with both coupling schemes can produce very narrow lines with linewidths down to $30 \mathrm{GHz}$. Further narrowing can be expected when cooled below $77 \mathrm{~K} .{ }^{15}$ The line center frequencies are precisely reproduced within the measurement accuracy of $1 \%$. The full width at half maximum (FWHM) linewidth measured for 1,2-DCB, TCNQ, and salicylic acid fit within the measurement accuracy of $10 \%$ to the previous values. ${ }^{7,3,16}$

\section{DISCUSSION}

In addition, the two different coupling schemes have to be compared under different aspects: Spectral coupling ratio, size of assembly, reproducibility, temperature stability, preparation time, and cost. As discussed above, the spectral coupling ratio of the flare is larger than the coupling using $\mathrm{Si}$

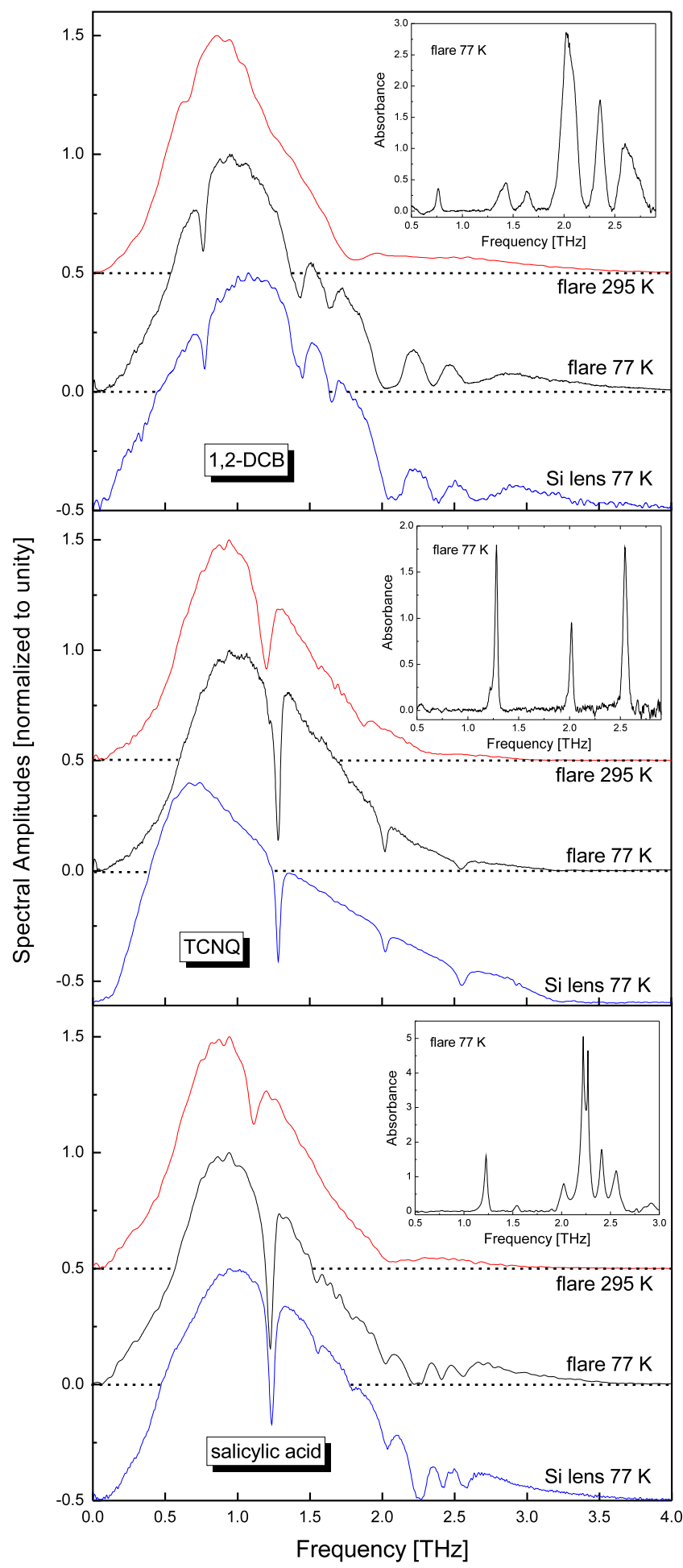

FIG. 7. (Color online) Spectral amplitudes for dropcast samples of 1,2dicyanobenzene (1,2-DCB), TCNQ, and salicylic acid (top-bottom) in the flare coupled WG at temperatures of at $295 \mathrm{~K}$ and $77 \mathrm{~K}$ compared to the results for the Si lens coupling. Insets: absorbance at $77 \mathrm{~K}$ using the flare coupled PPWG.

lenses. Especially when spectral features between $500 \mathrm{GHz}$ and $2 \mathrm{THz}$ are measured, the flare gives a significant increase in signal. Toward higher frequencies, the Si-PPWG performs nearly as well as the flared PPWG. This is to be expected as the metal's Ohmic loss increases for higher frequencies. This has more influence on the flare as the interface length between the metal and the $\mathrm{THz}$ beam is larger. 
TABLE I. Absorption features for dropcast samples (1,2-DCB, TCNQ, salicylic acid) measured with PPWGs using different coupling schemes (Si-optics vs metal flares) at a temperature of $77 \mathrm{~K}$. The detected line centers (FWHM line widths in parenthesis) are given in units of THz.

\begin{tabular}{|c|c|c|c|c|c|}
\hline \multicolumn{2}{|c|}{ 1,2-DCB } & \multicolumn{2}{|c|}{ TCNQ } & \multicolumn{2}{|c|}{ Salicylic acid } \\
\hline Si-PPWG ${ }^{\mathrm{a}}$ & Flare PPWG & Si-PPWG ${ }^{\mathrm{b}}$ & Flare PPWG & Si-PPWG ${ }^{\mathrm{c}}$ & Flare PPWG \\
\hline $0.77(0.04)$ & $0.76(0.04)$ & $1.28(0.03)$ & $1.28(0.03)$ & $1.23(0.04)$ & $1.22(0.04)$ \\
\hline $1.44(0.10)$ & $1.43(0.11)$ & $2.02(0.04)$ & $2.02(0.03)$ & $1.55(0.03)$ & $1.54(0.05)$ \\
\hline $1.67(0.06)$ & $1.63(0.07)$ & $2.56(0.07)$ & $2.54(0.05)$ & $1.79(0.06)$ & 1.80 (weak) \\
\hline $2.10(0.13)$ & $2.02(0.15)$ & & & $2.04(0.07)$ & 2.02 (satur.) \\
\hline $2.40(0.07)$ & $2.36(0.08)$ & & & $2.25(0.07)$ & $2.25(0.07)$ \\
\hline \multirow[t]{2}{*}{$2.68(0.19)$} & $2.60(0.17)$ & & & $2.42(0.04)$ & $2.41(0.05)$ \\
\hline & & & & $2.56(0.07)$ & $2.56(0.08)$ \\
\hline
\end{tabular}

${ }^{\mathrm{a}}$ Reference 7 .

${ }^{\mathrm{b}}$ Reference 3 .

${ }^{\mathrm{c}}$ Reference 16.

The reproducibility of the spectral features is very high for both WGs as seen in Fig. 7 and its evaluation in Table I. The line centers and line width are reproduced within the measurement accuracy. This is also to be expected since there is no fundamental difference between the two WGs for the interaction with the sample. Their geometric properties such as field extent and distribution are identical, except for the difference in type of coupling. In both WGs only the lowest order TEM-mode can propagate. ${ }^{8}$

Whether a line can be resolved is influenced by the spectral signal to noise ratio of the time-domain measurement, the scan length and the amount of applied sample (its absorption coefficient). For more sample in the WG, the integrated absorption is more intense. This is useful if weak absorption lines are investigated, but it leads to saturation for strong lines (see salicylic acid line at $2.02 \mathrm{THz}$ in Fig. 7). The high repeatability of the FWHM linewidth once again shows the high reproducibility of the crystal structure and good quality of the films while the accessible frequency range is the same for both WGs.

In terms of overall coupling values the experimental numbers are less than those obtained for an optimized flare. By working on parameters like flare length and curvature one could still get better performance. Depending on shape of the surface and input $\mathrm{THz}$ spotsize, further improvement to even higher values is expected. However, to be able to carry out measurements at $77 \mathrm{~K}$ temperature, it was necessary to limit the size of the flare assembly to a total length of $12 \mathrm{~cm}$. With a longer and thereby more adiabatic flare, even better results would be obtained.

The experiment showed that, due to the flexibility of the shim, the gap tends to be slightly smaller in the center due to the mechanical pressure of the spacers. This is not a problem as long as there is no direct contact between the two shims [see Fig. 2(b)]. For the presented coupling ratios this implies that the given values may underestimate the quality of the flare due to the possible narrowing of the gap. The gap can never be larger than the thickness of the spacers as the flares were mounted between the two rigid metal WG plates. To integrate this scheme into an independent sensor, mechanical end supports to mount the flare are advised.

This would also minimize the disadvantages of the free- standing flare for cryogenic temperatures. Due to the inherent bending of the shim and thermal inhomogeneities during the cool-down cycle, the flare slightly changes its shape. Therefore, the position of the flared PPWG had to be realigned to the center of the $\mathrm{THz}$ beam and slightly rotated (movement of the vacuum chamber) after the final temperature was reached. Comparable to our experience with the Si-PPWGs, the coupling of the flare also gets better with lower temperatures (most likely attributed to the reduced Ohmic losses in the metal ${ }^{18}$ ).

In terms of alignment the flare is much easier to install than a Si-PPWG, as no lens positions have to be aligned to form a line focus within the $50 \mu \mathrm{m}$ gap. So, the change between different samples is much faster. The material cost is also much less for the flares, as only low-cost copper shim is used. This is especially advantageous if one-time-use, dispensable sensors for industrial applications would be required.

\section{SUMMARY AND OUTLOOK}

We have shown that metal flares can be used to improve the coupling of linearly polarized free-space $\mathrm{THz}$ radiation into PPWGs. The coupling ratio at $1 \mathrm{THz}$ (35\%) for the adiabatic coupling compared to $22 \%$ using silicon lenses was improved by a factor of 1.6. The results are promising, as the new coupling scheme is easy to apply, uses simple components and does not require an alignment of Si lenses for each measured sample. Spectroscopic results of dropcast samples show that identical absorption features are detected for the two coupling techniques at both room and cryogenic temperatures. Comparable signal-to-noise ratios are also obtained for the two techniques.

\section{ACKNOWLEDGMENTS}

This work was partially supported by the Fraunhofer Gesellschaft FhG internal program for the scientific exchange (PROF. $x^{2}$ ) and the National Science Foundation.

\footnotetext{
${ }^{1}$ M. van Exter, C. Fattinger, and D. Grischkowsky, Opt. Lett. 14, 1128 (1989).
}

${ }^{2}$ R. A. Cheville and D. Grischkowsky, Opt. Lett. 23, 531 (1998). 
${ }^{3}$ J. S. Melinger, N. Laman, S. S. Harsha, S. Cheng, and D. Grischkowsky, J. Phys. Chem. A 111, 10977 (2007).

${ }^{4}$ M. Walther, B. Fischer, and P. Uhd Jepsen, Chem. Phys. 288, 261 (2003).

${ }^{5}$ D. Grischkowsky, S. Keiding, M. Exter, and C. Fattinger, J. Opt. Soc. Am. B 7, 2006 (1990).

${ }^{6}$ C. Konek, J. Wilkinson, O. Esenturk, E. Heilweil, and M. Kemp, Proc. SPIE 7311, 73110K (2009).

${ }^{7}$ J. S. Melinger, N. Laman, S. S. Harsha, and D. Grischkowsky, Appl. Phys. Lett. 89, 251110 (2006).

${ }^{8}$ R. Mendis and D. Grischkowsky, Opt. Lett. 26, 846 (2001).

${ }^{9}$ M. van Exter and D. Grischkowsky, IEEE Trans. Microwave Theory Tech. 38, 1684 (1990).

${ }^{10}$ J. Dai, J. Zhang, W. Zhang, and D. Grischkowsky, J. Opt. Soc. Am. B 21, 1379 (2004).
${ }^{11}$ Y. Zhao and D. Grischkowsky, IEEE Trans. Microwave Theory Tech. 55, 656 (2007).

${ }^{12}$ S.-H. Kim, E. S. Lee, Y. B. Ji, and T.-I. Jeon, Opt. Express 18, 1289 (2010).

${ }^{13}$ M. Theuer, R. Beigang, and D. Grischkowsky, Appl. Phys. Lett. 96, 191110 (2010).

${ }^{14}$ J. Zhang and D. Grischkowsky, Appl. Phys. Lett. 86, 061109 (2005).

${ }^{15}$ N. Laman, S. S. Harsha, D. Grischkowsky, and J. S. Melinger, Opt. Express 16, 4094 (2008).

${ }^{16}$ N. Laman, S. S. Harsha, and D. Grischkowsky, Appl. Spectrosc. 62, 319 (2008).

${ }^{17}$ S. S. Harsha and D. Grischkowsky, J. Phys. Chem. A 114, 3489 (2010).

${ }^{18}$ N. Laman and D. Grischkowsky, Appl. Phys. Lett. 93, 051105 (2008). 